\title{
EDITORIAL
}

\section{Anticancer Activity of Natural Compounds}

\author{
Abdul Q Khan, Shahab Uddin*
}

Asian Pac J Cancer Prev, 22, Anticancer Activity of Natural Compounds: HOW's on Methods and Reports Suppl, 1-2

Cancer, one of the leading cause of, socioeconomic burden and mortality in humans, accounting for approximately 9.6 million deaths worldwide yearly with future predictions suggest that by 2030, 13 million people will die from cancer each year (World Health Organization). There has been a lot of developments in exploring the underlying mechanisms of cancer pathogenesis in order to improve overall clinical outcomes of cancer patients and therapeutic complications including drug resistance. However, the overall burden due to cancer and associated challenges are increasing exponentially. Therefore, a deep and thorough investigation is required to get better cancer therapeutic drugs with reduced or minimal complications. Since ancient times, natural products are the prime source of therapeutics for various human diseases. Furthermore, natural compounds have been vital in drug development as they possess a range of biological activities and pharmacological features including their easy availability, less expensive and non-toxic action on normal cells that supports their potential as putative anticancer agent (Dutta et al., 2019). Moreover, it has been estimated that more than $70 \%$ of available anticancer drugs approved by United States Food and Drug Administration in the last few decades are either derived from natural resources or mimic them (Bishayee and Sethi , 2016). Natural compounds are the biologically active phytochemicals or phytonutrients present in plants and have enormous health promoting effects. These compounds can be classified according to the chemical structure as follows: terpenoids, carotenoids (lycopene, alpha and beta carotene), phenolic compounds - phenolic acids, flavonoids, stilbenes (resveratrol), coumarins, tannins; alkaloids, etc., are some of the potent category of phytochemicals with promising therapeutic potential against a number of chronic human diseases including cancer.

Recent updates indicate the multitarget potential of natural products that suggest their strong therapeutic potential against various complex human diseases including cancer and hence use of natural products with multitarget potential would be of great promising therapeutic value. Natural products have showed promising anti-cancer actions via targeting deregulated signaling mechanisms underlying cancer hallmarks including drug resistance, stemness features, metabolic reprogramming, epithelial to mesenchymal transition (EMT) and maintenance of tumor microenvironment. Interestingly, modulation of a number of signaling mechanisms including JAK/STAT3, PI3K/AKT, redox sensitive transcription factors like NF-Kb, AP-1, NRF2, etc, EGFR, mTOR, ATR,JNK, Notch, Wnt, Hedgehog, and MAPK, pathway, autophagy, apoptosis (IAP1, xIAP, Bfl-1/ A1, Bcl-2, cFLIP, and survivin), cell cycle proteins (cyclin D1 and c-Myc), and various other critical molecules associated with cancer pathogenesis by natural products provides strong basis for their anti-cancer potential. There has been various reports demonstrating how the bioactive molecules, derived from natural products, in combination with conventional chemotherapeutic agents enhance therapeutic outcome with minimal adverse effects (Namdeo et al., 2020; Lin et al., 2020). Generally, combination of natural products gives synergetic and or additive outcome and recently we have also showed that curcumin synergistically enhanced the anticancer action of cisplatin. Moreover, natural products also sensitize cancer cells to anti-cancer drugs which is another important mechanism relevant in achieving optimal cancer therapeutic outcomes with minimal adverse effects (Lin et al., 2020, Khan et al., 2020). Further combination of natural products with other drugs or inhibitors also provide positive therapeutic outcomes such as induction of apoptosis, attenuation of cancer drug resistance due to modulation of various underlying mechanisms. Use of herbal medicinal combinations or traditional Chinese medicines (TCM), highly prevalent through ought the world and particularly in China, has been shown to enhance survival and improve the quality of life of cancer patients (Lin et al., 2020). Natural products including flavonoids showed promising therapeutic outcome due to their pharmacological, antioxidant, anti-inflammatory and anticancer properties via modulating aberrant signaling mechanisms associated with tumorigenesis and cancer progression (Dutta et al., 2019, Bisol et al., 2020). Interestingly, these natural products also exhibit a range of features critical in cancer management such as anti-oxidant and anti-inflammatory potential and attenuation of the deregulated expression and functioning of non-coding RNA, epigenetic regulatory mechanisms, tumor microenvironment and multiple deregulated signaling pathways including JAK-STAT, PI3K-AKT, 
m-TOR, and EFR etc., critically associated with cancer hallmarks (Bisol et al., 2020, Anantharaju et al., 2016). In addition, these phytochemicals also showed promising therapeutic outcomes in chemoperception strategies, a method used to suppress or inhibit the multi-stage (initiation, promotion and progression) carcinogenesis due to accretion of genetic and epigenetic alterations by using natural and or synthetic products. Interestingly, there has been various clinical studies demonstrating importance of the chemopreventive action of natural products against different human malignancies (Jeter et al., 2019; Feng and Wang, 2012). In addition, natural products have also played vital role in managing the clinical complications due to cancer chemotherapy such as mucositis which are often very painful and lead to cessation of therapy and interestingly a number of clinical trials on phytochemicals have been done to manage the adverse toxic effects of conventional cancer therapeutic measures.

Overall, it can be said that natural products are the backbone for identification and development of therapeutic for various human ailments since long time. They have revolutionized the understanding of the mechanisms underlying disease pathogenesis including cancer. These compounds also vital in the development of combinational cancer therapeutics in order to achieve better clinical outcomes. Though enormous progress work has been made in developing clinically effective anticancer therapeutics from natural resources still we have not succeeded in translating the preclinical findings of plant based bioactive compounds in clinics at large scale and hence requires further attention in order to achieve our goals of developing novel and effective plant derived anticancer drugs.

\section{Acknowledgement}

The authors are thankful to Medical Research Centre, Hamad Medical Corporation, Doha, Qatar, for providing support (grant \#16354/16).

\section{References}

Anantharaju PG, Gowda PC, Vimalambike MG, Madhunapantula SV (2016). An overview on the role of dietary phenolics for the treatment of cancers. Nutr J, 15, 99.

Bishayee A, Sethi G (2016). Bioactive natural products in cancer prevention and therapy: Progress and promise. Semin Cancer Biol, 2016, 40-41:1-3.

Bisol A, de Campos PS, Lamers ML (2020). Flavonoids as anticancer therapies: A systematic review of clinical trials. Phytother Res, 34, 568-82.

Dutta S, Mahalanobish S, Saha S, Ghosh S, Sil PC (2019). Natural products: An upcoming therapeutic approach to cancer. Food Chem Toxicol, 128, 240-55.

Feng L, Wang Z (2012). Clinical trials in chemoprevention of head and neck cancers. Rev Recent Clin Trials, 7, 249-54.

Jeter JM, Bowles TL, Curiel-Lewandrowski C, et al (2019). Chemoprevention agents for melanoma: A path forward into phase 3 clinical trials. Cancer, 125, 18-44.

Khan AQ, Mohamed EAN, Hakeem I, et al (2020). Sanguinarine induces apoptosis in papillary thyroid cancer cells via generation of reactive oxygen species. Molecules, 25.

Lin SR, Chang CH, Hsu CF, et al (2020). Natural compounds as potential adjuvants to cancer therapy: Preclinical evidence. Br J Pharmacol, 177, 1409-23.

Namdeo AG, Boddu SHS, Amawi H, et al (2020). Flavonoids as multi-target compounds: A special emphasis on their potential as chemo-adjuvants in cancer therapy. Curr Pharm Des, 26, 1712-28.

The L. GLOBOCAN (2018). Counting the toll of cancer. Lancet, 392, 985. 